\title{
ACCESSIBLE ROBOTS FOR IMPROVING SOCIAL SKILLS OF INDIVIDUALS WITH AUTISM
}

\author{
Matthew F. Tennyson ${ }^{1}$, Deitra A. Kuester ${ }^{2}$, John Casteel $^{1}$, Christos Nikolopoulos ${ }^{3}$ \\ ${ }^{1}$ Department of Computer Science and Information Systems \\ Murray State University, Murray, KY, USA \\ ${ }^{2}$ Teacher Education, Bradley University, Peoria, IL, USA \\ ${ }^{3}$ Departments of Computer Science \& Information Systems \\ Bradley University, Peoria, IL, USA
}

\begin{abstract}
This paper reports on an ongoing project between members of the computer science and special education departments of Bradley University and Murray State University, detailing the robotic platforms developed and investigated as a potential tool to improve social interactions among individuals with Autism Spectrum Disorders (ASD). Development of a fourth generation robotic agent is described, which uses economically available robotic platforms (Lego NXT) as Socially Assistive Robotics (SAR), combined with direct instruction pedagogy and social scripts to support an alternative educational approach to teaching social behavior. Specifically, in this fourth generation, changes to the physical design of the robots were made to improve the maintainability, reliability, maneuverability, and aesthetics of the robots. The software architecture was designed for modularity, configurability, and reusability of the software.
\end{abstract}

Keywords: Autism Spectrum Disorders (ASD), Socially Assistive Robotics (SAR), Telerobotics, Telemanipulation, Social Skills

\section{Introduction}

According to the Centers for Disease Control and Prevention, one in every 68 children (1:42 boys, 1:189 girls) has Autism Spectrum Disorder (ASD) [1]. Individuals with ASD exhibit impairments in three key areas: (a) communication, (b) social interaction, and (c) restricted interests and repetitive behaviors. The American Psychiatric Association [2] recently redefined qualifiers for ASD, citing levels of severity, the impact deficits key areas have on the quality of life and the amount of support needed, beginning with Level I (less support, formerly included diagnosis of Asperger Syndrome, Pervasive Developmental Delay-Not Otherwise Specified), Level II (moderate support), and
Level III (most support).

Children with ASD exhibit their impairments in unique combinations. These impairments typically include notable developmental delays and or deficits in comparison to the child's neurotypical peers, which can include both speech and behavioral issues that affect social interaction. Many parents and care providers of individuals with autism say that, "If you've met one person with autism, you've met one person with autism," meaning that each individual with ASD displays symptoms and impairments in a way that are essentially unique to that individual (as opposed to the phrase "if you've seen one, you've seen them all"). This often results in the need for individual-specific treatment, 
as different individuals react to the same treatment in different ways.

These deficits and/or delays inhibit peer relations and increase peer rejection [3]. For individuals who exhibit poor social skills, outcomes for independent living and a productive quality of life into adulthood are hampered by unstable relationships, problems with the legal system, mental health conditions [4-7], an inability to attain or maintain competitive employment, and experience an overall poor quality of life [8]. In one report that reviewed 16 studies, all of the 398 adults (age $>18$ ) who participated had an overall quality of life that was poor [8]. Moreover, since the lack of social skills seems to be a contributing factor to the poor quality of life, it is increasingly important for individuals with ASD to learn appropriate social behavior during their school experience. The challenge is, however, how to teach appropriate behavior while simultaneously sustaining motivation and attention.

As previously stated, there is no single established standard treatment for individuals with ASD. However, individuals with ASD have shown improved social competencies through the use of evidence-based teaching strategies, such as repetition, imitation and role-play [9-12]. A variety of other evidence-based social communication approaches and interventions have also been used to improve the social deficits among individuals with ASD including: Applied Behavior Analysis (ABA); communication interventions such as social stories or social scripts (play-acting realistic social situations, such as personal space, joining groups, saying hello and other common interactions); and direct instruction (teacher-directed instruction that uses sequential steps toward goal attainment) [9-13].

Repetition and familiarity are common components of these interventions, which may help to explain why these interventions are successful among individuals with ASD. Despite repetition and familiarity, one cannot ignore the inherent unpredictable human-to-human interaction necessary in using these interventions, thereby creating potential obstacles for skill acquisition among individuals with ASD.

Thus, a challenge that continues to confront teachers is how one can teach and promote social relationships among individuals with ASD when repetition and familiarity support success, but unpre- dictable human interaction (teacher and peers alike) negates repetition and familiarity during the teaching and learning process. Reducing the amount of unpredictable human-human interaction during the teaching phase may be an alternative solution to this problem, and using a predictable robot as a social catalyst through which social behavior may be taught seems viable.

\section{Using Socially Assistive Robotics as an Intervention for Individuals with Autism}

Several technology-based approaches have been used to teach social skills to individuals with ASD. For example, video instruction has been used, a survey and review of which is provided in [14]. Evidence has been presented that virtual peers (in the form of interactive animations) more effectively engage children with ASD in contingent discourse than do human counterparts [15-16].

In comparison to these technology-based approaches to teach social skills, Socially Assistive Robotics (SAR) allow a child to physically interact with a three-dimensional object through touch, natural language communication, physical play (e.g. hide and seek [17] and blowing bubbles [18]), and proximity, versus two dimensional interactions using a computer screen or life-size child image projected on a wall or flat surface. Unlike video instruction, SAR not only provides the opportunity to learn from a nonthreatening, three-dimensional inanimate object, it also presents the opportunity to learn through imitation and interaction thus encouraging autonomous social behavior [19]. The use of SAR has helped to decrease stereotypic behavior among individuals with ASD when compared to human-human interactions [20] and has improved communication of the same [20-21].

SAR can also be made responsive to changing conditions in their immediate environment to help support flexibility in applied behavior and individual responses of the child using Artificial Intelligence (AI). This is largely dependent on the software used by the SAR and any hardware limitations such as available sensors and processing components. Even with limitations, however, scripts provided by researchers or therapists can modify the 
actions of the SAR to drive it through a series of actions or recordings that are unique to the individual session. This mobility and versatility of the teaching tool may positively affect and encourage autonomous behavior.

Moreover, SAR are motivating, interesting, and engaging, which is validated in the research worldwide documenting positive outcomes in using SAR for therapeutic and rehabilitative purposes. We have also shown promise in using SAR as evidenced by the observed behavior among students during preliminary trials within the school and home environment $[22-24,34]$. As a result, it is widely believed that SAR may hold significant promise for behavioral interventions of those with ASD. Acting as a social agent through which appropriate behavior may be taught to individuals with moderate to high functioning ASD, SAR will require minimal human (teacher) interaction during the teaching phase, as well as predictable behavior using preprogrammed social scripts.

A child with ASD who works with SAR in the therapeutic setting may display emotions and levels of interaction that are not seen outside of that setting. One child, a 12-year-old named Louie, was observed asking questions of the investigator running the SAR session. This included questions such as "Did you bring the robots today? Can I play with the robots today? Can I hold it?". The questions continued, expressing not only a desire to play with the robots but also a genuine interest in them: "Did you make these? Can I turn it on? Can I touch it?". While these questions may seem normal for a 12-year-old boy, in Louie's case most of his speech came in the form of scriptingthe incessant repetition of select words, phrases and dialogue from sources such as television shows, songs and movies. However, when SAR was introduced, Louie stopped scripting and displayed neverbefore-seen conversation-like behavior. [34]

Previous research has focused on SAR interventions within therapeutic/clinical environments [25-31]. For a comprehensive review of these approaches, refer to [32]. Robots used in these studies are expensive, in most cases are not available to consumers, and may be complicated to program and use. There is a need for robotic platforms which are less costly and allow greater access, ease of acquisition, programming and practi- cal use. Therefore, a variety of combined SAR and social scenarios within a variety of natural environments (school/college, home, community) are being explored in this project. For a description of other robotics platforms that have been explored, refer to [22-24, 34]. Target behaviors include social skills that promote autonomous behavior (e.g. personal space, touching others' things without asking, initiating conversation, etc.).

\section{Previous Generations and Field Tests}

In earlier studies [22-24, 34], robotic agents were successfully utilized that were built by modifying the Lego NXT robotic platform and the leJOS software development environment. These prototypes were referred to as the first, second, and third generations of robots. The Lego NXT platform was chosen as it satisfied the necessary requirements to be effectively utilized in the ASD application domain, as outlined in [22-23, 34]. The reader is referred to the earlier papers for a description of the developed robotics platforms and related technologies.

Louie, the 12-year-old child previously mentioned, was one of the children who worked with some of the Lego robots created for these studies. In addition to bringing out non-scripted speech used for personal interaction, the robots assisted him in learning lessons on social topics such as personal space. The SAR were able to elicit responses from him that human therapists and workers could not, so it is beneficial for the robots to be as interactive and customizable to the needs of the individual therapy recipients as possible. This is a driving reason behind the evolution of the robots across the generations, as each generation provided additional options for researchers and therapists to customize the scripts and interactions that the robots provided.

The first generation robots were humanoid robots that simply recited parts of speech that could potentially be used as part of a social script ("Hi", "Good-bye", "Have a nice day"); these were included in the pre-packaged consumer Lego kit.

The second generation included several changes to the robots to address the issues encountered with the first generation. The physical part of the social 
script involved two actors and hence required precision and coordination in movement and speech of the robots to simulate a two-way social interaction between robots. A wheel assembly replaced legs in order to allow the robots to move more accurately and faster. The robots were still limited in their speech capacity due to the NXT brick's available memory and used a timing system to determine when each robot should act, which resulted in difficulty keeping the robots properly synchronized.

The third generation of robots was thus developed with the robot frame completely redesigned with tracks to improve accuracy of movement. A gearing system was added to the tracks so that the motors could operate at a slower speed and thus produce less noise, as some of the human subjects showed sensitivity to the noise of the rotating motors. Because the original NXT brick sound quality and volume was not adequate to make the audio suitable in real-world environments that included a variety of background noises, the bricks' internal speakers were replaced with external audio jacks and external speakers were plugged into the audio jacks and mounted on the robots.
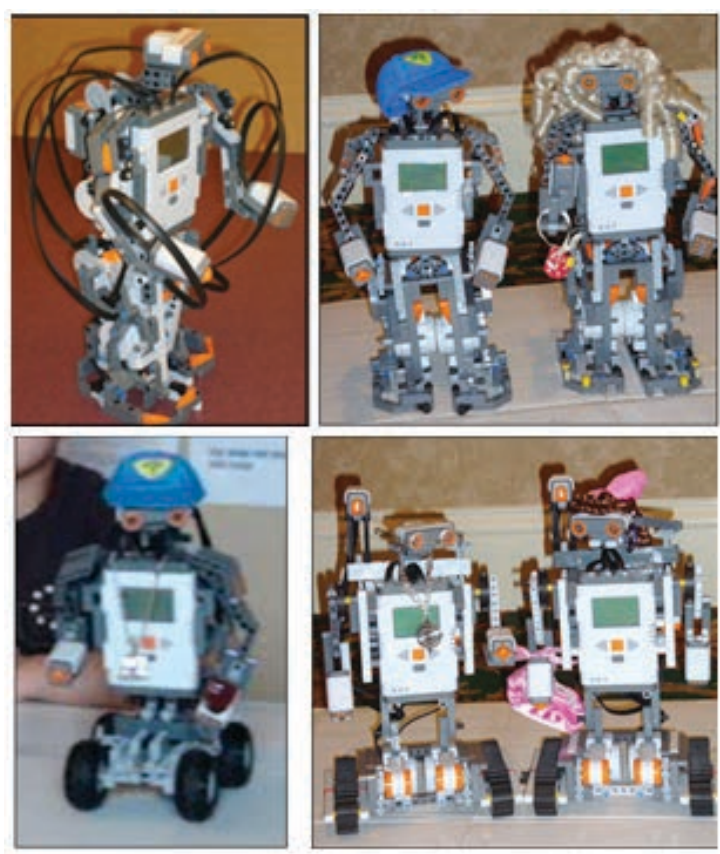

Figure 1. Generational modifications of robotic agents. From top-left to bottom-right: "Alpha

Rex" instructions for which are included with the Lego Mindstorms NXT kit, 1st generation "Rex and Alphena", 2nd generation "Robbie", and 3rd generation "Johnny and Julie."
The software was also redesigned to utilize telerobotics to address the limited storage capacity of the NXT bricks. Control instructions and sound files were sent from a laptop computer via Bluetooth to the two robotic actors as appropriate. Telerobotics not only extended the capability to control the robot and send information to it, it also allowed for controlling two or more robots from a single laptop computer.

Preliminary field tests of this project included gradual introduction to modified Lego NXT platforms (see Figure 1), which were used to help gauge participant response, motivation and sensory thresholds. Each interaction involved the use of at least two robots playacting preprogrammed social scripts. Louie and two other participants interacted with the same robots and all of the robots were preprogrammed with the same social scripts which dealt with invading personal space and touching others' things without asking.

The children responded positively, though they did display different responses. While Louie excitedly asked questions and took an active interest in the robots being set up, Dorothy, a nonverbal high school senior with ASD watched the investigator set up the robots while rocking back and forth as a form of comforting self-stimulation, which she typically did during unfamiliar situations. Prone to violent outbursts when changes occurred in her daily schedule, Dorothy instead, stopped rocking and appeared to tentatively watch the robotic interactions by looking in the direction of the robotic display (versus looking around the room or at others). Despite the changes in her schedule whenever she interacted and or observed the robots, she never displayed a violent outburst, refrained from self-stimulating movement, and smiled and giggled whenever the robots spoke. Similar to Louie, this was never-before observed behavior.

While preliminary data and teacher and parent surveys helped to guide the selection of overlapping target behavior among participants and subsequent development of social scripts, one may ask how the same social script could be appropriate across individuals when no two individuals with ASD are alike. Even in the examples of Louie and Dorothy, one can see that both of the children's behaviors and their reactions were significantly different despite the use of the same robots using the same script. 
Understanding that ASD is a spectrum of disorders, individuals identified exhibit an array of behaviors with varying degrees of severity. Although there are varying degrees of severity and an array of behaviors, overlapping deficits exist in three key areas and it is within these three key areas consistent behavioral deficits may be observed. Typical deficits among this population of learner include a lack of appropriate understanding about personal space and touching others things without asking first, both of which were evidenced among all participants during baseline observations of preliminary trials, as well as reported by teachers and parents. Therefore, to support reliability of the intervention across individuals and environments, the same social scripts and the same robots were used.

Social competencies are crucial to both academic and life success, as both school and day-today activities require a good deal of interaction with other people. Unfortunately, children with ASD participate significantly less in social, recreational and leisure activities, and have significantly fewer friends (including having a "best friend") than those with other intellectual disabilities or their neurotypical peers [33]. This creates challenges for teachers, therapists and parents in that the teaching phase necessitates human interaction, as do general peer relations. The challenge that the SAR and their scripts hoped to meet is overcoming the difficulty that is encountered while teaching appropriate behavior, simultaneously minimizing the need for selfstimulation during an unfamiliar situation and unpredictable behavior during the teaching phase of skill acquisition.

Field tests included social scripts that addressed the selected issues, with the scripts increasing the duration of robot-robot interaction over time. Robots playacted their way through the social interactions while participants observed. Two of the three interactions involved a social script with embedded comprehension questions about the interaction and statements directed toward the participants, eliciting human-robot interaction (HRI) and gauging both the subject's comprehension of the script and how well the script met its intended purpose of using robots as teachers.

Quantitative analyses of preliminary field tests included the duration of perceived eye gaze (looking in the direction of the robots throughout the in- teraction, sustained attention in looking in the same direction of the robots, looking at the robots [in the same direction] when each the robot spoke), perceived tracking (head movement as if they were following the robots [or in the same direction] as the robots moved, turning head in the direction of the robot as it moved) and the number of correct comprehension questions answered post-interaction. A positive correlation emerged between robot-robot interaction, perceived eye gaze and tracking. Eye gaze and tracking increased simultaneously with the length of each social script (see Figure 2). Participants seemingly watched the robots (or faced the respective robot when it spoke or moved) throughout each interaction, regardless of the duration of the interaction or movement of the robot, and correctly answered questions about what the robots said and to whom they believed the robot was speaking.

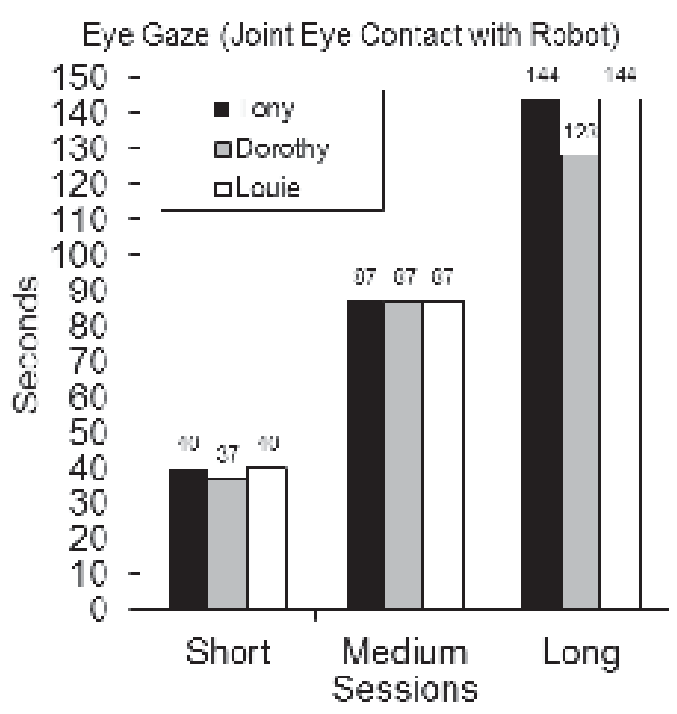

Figure 2. Results measuring eye gaze with gradual increase of exposure to robots programmed to simulate invasion of personal space during preliminary investigative trials of 40 seconds, 87 seconds, and 144 seconds among three children with ASD.

Qualitatively, ad hoc behaviors were continuously monitored with each modification made to the platforms. These behaviors included: anticipated challenges when routines were interrupted or when unfamiliar objects or people were introduced versus typically being aloof to these types of changes or stimuli; violent outbursts or temper tantrums when unexpected changes were imposed; moving closer or away from the robots (or the ta- 
ble upon which the robots interacted) before, during or after each interaction; initiating conversation (regardless who was in the room or with whom s/he initiated during pre- and post-interaction) versus responding only when prompted or not at all; and reduced/increased scripting, rocking or other forms of self-stimulation. All of these ad hoc behaviors were observed baseline types of behavior, allowing researchers to accurately note when observable changes occurred within the individual subjects.

Sustained attention, interest and motivation were evident among participants. This took different forms in different participants, as illustrated in both Louie's questions and Dorothy's laughter and focus as mentioned above. There were no adverse effects noted as a result of using robots as social agents for the participants.

\section{The Fourth Generation}

In the current (fourth) generation, both the physical design and the software design changed significantly. In regards to the physical design, there were two major updates: (1) the brick was positioned so that it could easily be accessed and (2) the tracks were replaced with wheels and a caster. In regards to the software design, the software was re-structured to support a modular client-server architecture. Finally, the traditional speakers were replaced with wireless Bluetooth speakers. Each of these changes is described in further detail in the remainder of this Section. See Figure 3 for a visual comparison of the two latest generations of robots.

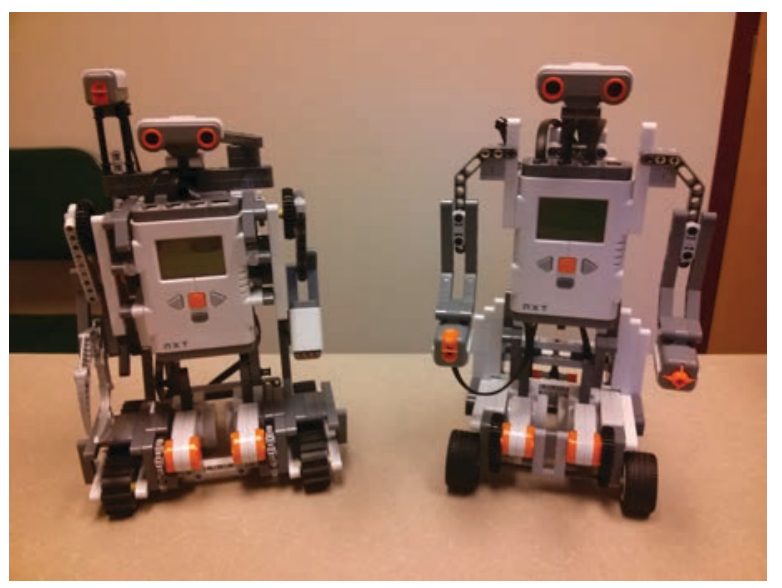

Figure 3. Visual comparison of third and fourth generations

\subsection{The Physical Design}

As previously noted, the physical design of the robot was changed by repositioning the brick. The placement of the brick was indeed a major deficiency in the design of the third generation. Replacing the battery pack or the brick itself required major disassembly of the robot due to the "wraparound" nature of the back and arm assembly. The robot was connected to the brick in numerous places along the back and both sides of the brick itself. The assembly of the back of the robot and its arms was overly complex. See Figure 4 for a comparison of the third and fourth generation arm assemblies.

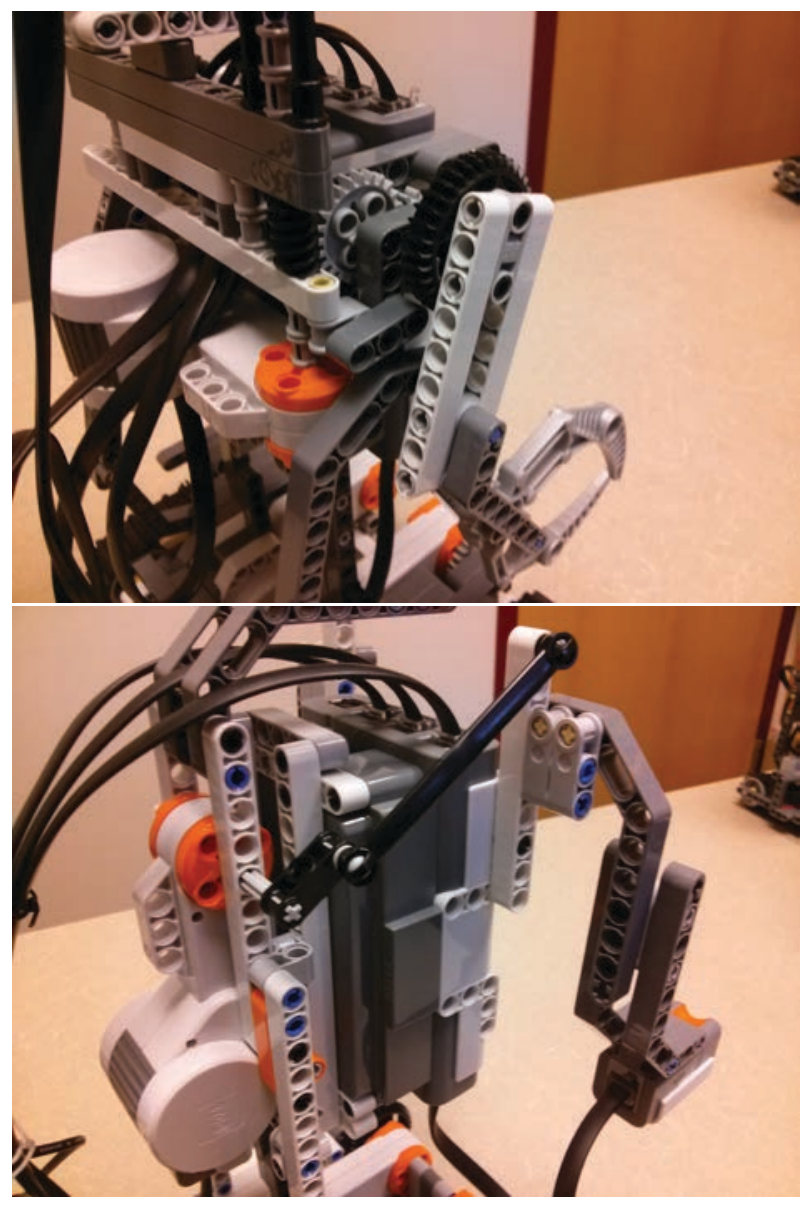

Figure 4. Comparison of third and fourth generation arm assemblies

As can be seen in Figure 4, the third generation arm assembly required a complex gearing system that incorporated four gears, including a worm gear that converted rotational motion about one axis into rotational motion about a perpendicular axis. As can be seen in the Figure, the design was drastically simplified in the fourth generation by elimi- 
nating all gears and by reducing the number of connecting points down to a single point of connection. These changes improved the maintainability, reliability, and aesthetics of the robots.

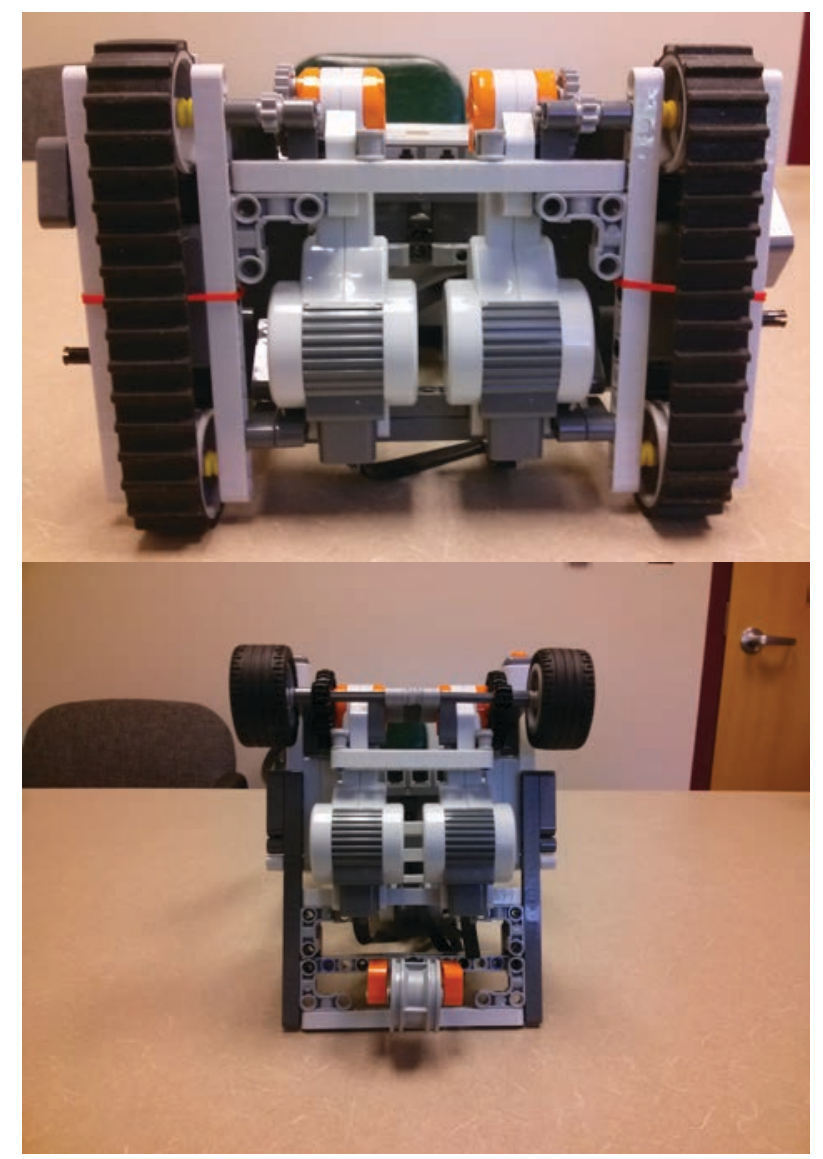

Figure 5. Bottom view comparison of third and fourth generation

The physical design of the robot was also changed by replacing the tracks with wheels (see Figure 5). More precisely, the tracks were replaced with two driving wheels and a caster. The tracks caused bumpy movement and difficulties steering due to the skidding of the tracks. Tracks are not necessary in this application domain in which the robots typically move upon a smooth and level surface, such as a tabletop or floor, where traction is not a significant factor. Note that tracks were used to replace wheels in a previous generation. In the previous generation, however, four wheels were used, none of which pivoted. Tracks were used at that time to improve maneuverability, which they indeed did. However, they also resulted in jerkier movement. Using two wheels with a caster combined the benefits of both previous generations: smooth movement and excellent maneuverability.

\subsection{The Software Design}

The software architecture is perhaps the most novel aspect of the fourth generation robots. The software is extremely flexible and modular, and was designed with telemanipulation at the forefront. There are three major software components: the NXTRobot, the PCRobot, and the Script.

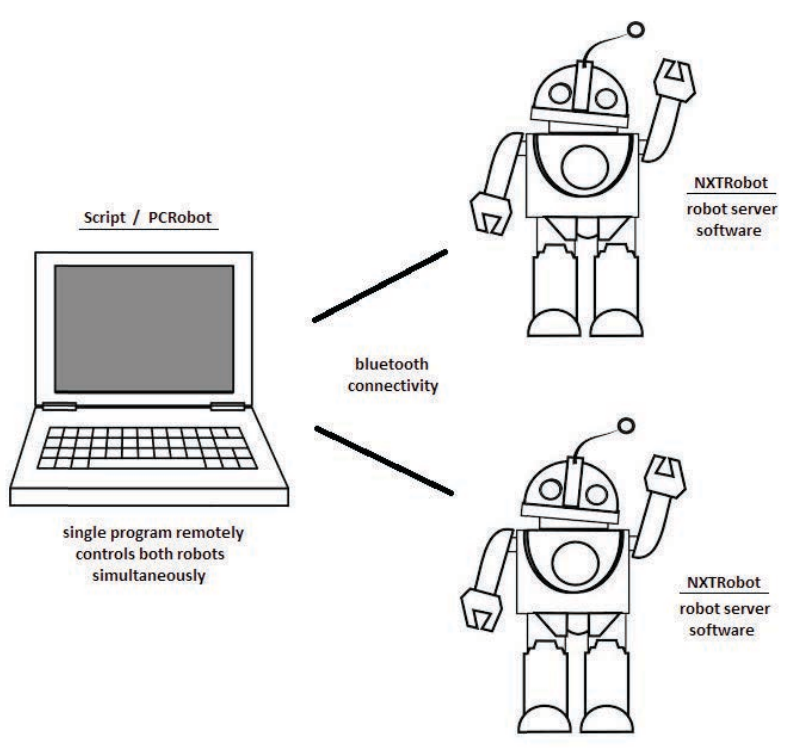

Figure 6. Basic system architecture

The NXTRobot is a sever program that is executed onboard the robot by the leJOS virtual machine. Upon start-up, this software waits for a Bluetooth connection. After a connection is made, it waits for commands via Bluetooth and when received acts upon those commands. A protocol is defined for streaming the commands to the robot via the Bluetooth connection. The commands include fundamental instructions, such as DRIVE_MOTOR_A, STOP_MOTOR_A, SET_LIGHT, SET_ATTRIBUTE, READ_INPUT, DISCONNECT, etc. The software is designed to work on literally any NXT robot. Therefore, the software running on the robots themselves never has to be updated. There is no intelligence built into the onboard software itself. The robot acts as a dumb terminal that receives commands, acts upon them, and returns data when applicable.

The PCRobot is a software library that executes on the controlling laptop. The PCRobot implements the protocol for sending commands to the NXTRobot via Bluetooth. It is also extremely config- 
urable. It allows the application (a.k.a., the Script, which will be discussed next) to describe the physical attributes of the robots. For example, the application will specify the wheel radius, the wheel base, which motor controls each wheel, and the rotational direction of the wheels relative to the motors. The PCRobot provides an API to allow the application to turn the robot to the right or left at a specified speed and turning radius. Based on that request and the respective physical configuration, the PCRobot library then calculates exactly which motors need to turn at what speed and duration. The corresponding commands are sent to the NXTRobot server software via Bluetooth, and the robot presumably complies.

The Script is simply an application that remotely controls one or more robots. In our particular application domain, the Script usually includes a simple social script meant to help demonstrate social skills to individuals with ASD. For example, two robots will approach each other, verbally introduce themselves, exchange niceties, say goodbye, and finally depart. The Script will instantiate the necessary number of PCRobot objects (one for each physical robot), specify the physical attributes for each robot, then proceed with the social script by specifying movements and speech. However, the Script could include more complex logic and implement interesting AI, based on the specific needs of the application and purpose of the robots themselves.

This software architecture is extremely modular, configurable, and reusable. The NXTRobot server software should never have to be updated, once flashed onto a robot, and is capable of controlling all of the robot's fundamental tasks. The PCRobot library is extremely configurable, and makes few assumptions about the type of robot it is telemanipulating. It hides all of the "gory details" regarding motors, sensors and other devices from the application. The Script simply implements the application to fulfill its purpose, with the PCRobot providing the tools that it needs to do so easily.

\subsection{The Sound System}

In the third generation, the factory Lego NXT bricks were manually modified by replacing the bricks' internal speakers with external audio jacks. External speakers were then plugged into the audio jacks and mounted on the robots. This was necessary because the original NXT Brick sound quality and volume was not adequate.

However, this approach became problematic for a variety of reasons. First, the manual modification of the bricks was neither robust nor reliable. The modifications required drilling into the factorymade brick, removing the internal speaker, and replacing it with an audio jack by mounting it and soldering the electrical connections. These manual modifications were not as reliable as the original production-quality product and degraded relatively quickly over time.

Second, sound had to be streamed to the robot via the Bluetooth connection due to the limited memory onboard the NXT brick. Having to store sound files onboard the robots would also deviate from the architecture outlined in the previous Section. Because sound had to be streamed in this manner, other commands were blocked as the sound files were streamed, and the sounds were limited to specific sampling rates due to the streaming and playback mechanism. This directly affected the quality of the scripts.

Due to these limitations, the sound system was revamped in the fourth generation. The external speaker was replaced with a Bluetooth speaker, eliminating the need to modify the factory NXT brick. Each robot now carries its own Bluetooth speaker. The laptop is paired with each speaker as usual with any Bluetooth device. When a robot needs to emit a sound, its respective Bluetooth speaker is activated, and the sound is played as normal. This eliminates any coupling between the sound files (which are application-specific) and the robots (which execute dumb server software and should, therefore, remain expressly applicationneutral). It also eliminates the issue related to blocking commands while sound is streaming. A new process is spawned when a sound should be played and the correct speaker is activated, and the operating system then manages the execution of that process. The script performed by the robot proceeds with execution as normal, unimpeded by the process that the operating system uses to play the sound file. 


\section{Conclusion and Future Work}

It is a major challenge for teachers, parents, and service providers of individuals with ASD to teach social skills to individuals who may struggle with unfamiliar, unpredictable human interaction. In this paper, we described the ongoing development of a fourth-generation economical, consumeravailable robotic platform and results of subsequent field tests using SAR as an educational intervention, combined with direct instruction pedagogy and target-behavior social scripts. Using this fourthgeneration model, we intend to move forward with field tests where the "actors" and scripts target specific social skill deficits within home and community environments. Data collected from these tests will not only allow us to continue documenting the effectiveness of the model but may also assist with improvements to the NXT-based SAR in future generations.

Children with ASD exhibit deficits in a variety of social behavior, resulting in peer rejection and an overall poor quality of life. Children with ASD are affected by this more than children with any other developmental disability [8-9, 33]. Their increased perceived anxiety creates a greater perceived need for arousal (self-stimulation), especially in unfamiliar situations, and this contributes to a continuous pattern of stereotypic behavior, avoidance, and rejection. SAR can have a significant impact on breaking this pattern [20-21], and the fourthgeneration model of economical SAR using the Lego NXT platform could put the technology in reach of a much wider number of children who need it.

Future studies will utilize single case methodology to ensure social validity (using pre-study interviews/discussions with parents and baseline behavioral observations), treatment fidelity (procedural integrity checks, comprehension questions of participants post-intervention, training of users of robots) and inter-observer reliability (comparison of data between researchers, observers and therapists). Baseline will include previous unsuccessful interventions used to help teach participants appropriate social behavior, observations, interviews, and the number of times the student exhibits (or should have exhibited) target behavior (e.g. invasion of personal space, joining a group of peers, etc.). Post- intervention data and maintenance phases will be collected and analyzed using multiple sources of triangulated evidence (e.g. observation, interviews, antecedents, consequences, inter-rater agreement and other forms of data collected during sessions with the SAR.)

In future generations, the robots could be designed with more complex and sophisticated set of intelligent behaviors, such that the robot is not given a priori global knowledge of the environment. Instead, it would use its available sensors to construct a dynamic model of its environment by sensing locally as it progresses through the environment, which could change in unpredictable ways. The robot could also be modified to include a camera, microphone or other recording devices to record data and allow an operator to view the interaction from the robot's perspective. A screen or other interface device could potentially be mounted on the robot to allow for two-way communication between the robot and the individual with ASD. Finally, a Graphical User Interface (GUI) could be created allowing educators and parents to create their own customized social scripts for the robots to perform, based on their student or child's individual needs.

\section{References}

[1] J. Baio, Prevalence of Autism Spectrum Disorders: Autism and Developmental Disabilities Monitoring Network, 14 Sites, United States, 2008, Morbidity and Mortality Weekly Report Surveillance Summaries, 2012, vol. 61, no.3.

[2] American Psychiatric Association. Diagnostic and Statistical Manual of Mental Disorders, 5th Edition (DSM-5), American Psychiatric Publishing, 2013.

[3] I. Werry, K. Dautenhahn, B. Ogden, W. Harwin, Can Social Interaction Skills Be Taught by a Social Agent? The Role of a Robotic Mediator in Autism Therapy, Cognitive Technology: Instruments of Mind, vol. 2117 Lecture Notes in Computer Science, 2001, pp. 57-74.

[4] G. Barnhill, Outcomes in adults with Asperger Syndrome. Focus on Autism and Other Developmental Disabilities, 2007, vol.22, pp.116-126.

[5] I. Engstrom, L. Ekstrom, B. Emilsson, Psychosocial functioning in a group of Swedish adults with Asperger Syndrome or high functioning autism. Autism: International Journal of Research and Practice, 2003 pp. 99-110. 
[6] B. Hofvander, R. Delorme, P. Chaste, A. Nydn, E. Wentz, O. Sthlberg, et al. Psychiatric and psychosocial problems in adults with normalintelligence autism spectrum disorders. BMC Psychiatry, 2009, pp. 9-35.

[7] P. Howlin, S. Goode, J. Hutton, M. Rutter, Adult outcome for children with autism. Journal of Child Psychology Psychiatry, 2004, vol. 45, is.2, pp. 212-229

[8] H. Chiang, I. Wineman, Factors associated with quality of life in individuals with autism spectrum disorders: a review of the literature. Research in Autism Spectrum Disorders, 2014, vol.8, pp. 974986

[9] S. Greenspan, S. Wieder, Developmental patterns and outcomes in infants and children with disorders in relating and communicating: A chart review of 200 cases of children with autistic spectrum diagnoses. Journal of Developmental and Learning disorders, 1997, vol. 1, pp. 87-141.

[10] C. Gillberg, Asperger syndrome and high functioning autism. British Journal of Psychiatry, 1998, vol. 172,pp. 200-209.

[11] F. Volkmar, A. Klin, D.Cohen, Diagnosis and classification of autism and related conditions: Consensus and issues. Handbook of autism and pervasive developmental disorders, vol. 2, 1997, pp.540 .

[12] O. Grynszpan, J. Martin, J. Nadel, Exploring the influence of task assignment and output modalities on computerized training for autism. Interaction Studies, 2007, vol. 8(2), pp. 241-266.

[13] D. Baer, M. Wolf, R. Risley, Some current dimensions of applied behavior analysis. Journal of Applied Behavior Analysis, 1968, vol.1, pp. 91-97.

[14] S. Shukla-Mehta, T. Miller, K. Callahan, Evaluating the Effectiveness of Video Instruction on Social and Communication Skills Training for Children With Autism Spectrum Disorders: A Review of the Literature. Focus on Autism and Other Developmental Disabilities Journal, 2010, vol. 25, no. 1, pp. 23-36.

[15] A. Tartaro, Storytelling with a virtual peer as an intervention for children with autism: assets doctoral consortium. In: The Seventh International ACM SIGACCESS Conference on Computers and Accessibility, Baltimore ACM Issue 84, 2006, pp. 4244.

[16] A. Tartaro, J. Cassell, Playing with Virtual Peers: Bootstrapping Contingent Discourse in Children with Autism. ICLS'08 Proceedings of the 8th international conference on International conference for the learning sciences - vol. 2, 2008, pp. 382389.

[17] J. Trafton, S. Trickett, A. Stitzlein, L. Saner, C.D. Schunn, S.S. Kirschenbaum, The relationship between spatial transformations and iconic gestures. Spatial Cogntion and Computation, 2006, vol. 6(1), pp. 1-29.

[18] F. Michaud, J.F. Laplante, H. Larouche, A. Duquette, S. Caron, D. Letourneau, P. Masson, Autonomous spherical mobile robot for child development studies. IEEE Transactions on Systems, Man and Cybernetics, 2005, vol. 35, pp. 471-480.

[19] B. Robins, K. Dautenhahn, R. Boekhorst, and A. Billard, Robotic assistants in therapy and education of children with autism: can a small humanoid robot help encourage social interaction skills? Universal Access in the Information Society, 2005, vol. 4(2), pp. 105-120.

[20] I. Luthffi, S. Syamimi, Y. Hanafiah, A. Fazah, Z. Nur Ismarrubie, Robot-based Intervention Program for Autistic Children with Humanoid Robot NAO: Initial Response in Stereotyped Behavior. International Symposium on Robotics and Intelligent Sensors 2012 (IRIS 2012), Procedia Engineering, vol. 41, 2012, pp. 1441-1447.

[21] B. Huskens, R. Verschuur, J. Gillesen, R. Didden, E. Barakovak, Promoting question-asking in school-aged children with autism spectrum disorders: Effectiveness of a robot intervention compared to a human-trainer intervention, Developmental Neurorehabilitation, pp. 1-12, 2012.

[22] C. Nikolopoulos, D. Kuester, M. Sheehan, S. Dhanya, Investigation on Requirements of Robotic Platforms to Teach Social Skills to Individuals with Autism, vol. 59 Lecture Notes of the Institute for Computer Sciences, Social Informatics and Telecommunications Engineering, 2011, pp. 6573.

[23] C. Nikolopoulos, D. Kuester, M. Sheehan, D. Sneeha, W. Herring, A. Becker, L. Bogart, Socially assistive robots and autism, Journal of Solid State Phenomena, 2010 , vol. 166-167, pp. 315-320.

[24] C. Nikolopoulos, D. Kuester, M. Sheehan, S. Ramteke, A. Karmarkar, S. Thota, J. Kearney, C. Boirum, S. Bojedla, A. Lee, Robotic Agents used to Help Teach Social Skills to Children with Autism: The Third Generation. In proceedings of the IEEE International Symposium on Robot and Human Interactive Communication (RO-MAN), Atlanta, 2011, pp. 253-258.

[25] K. Dautenhahn, Robots as social actors: Aurora and the case of autism, in: Proceedings of the Third 
Cognitive Technology Conference, San Francisco, vol. 359, 1999.

[26] D. Feil-Seifer, M. Matari, Defining socially assistive robotics. In proceedings of the International Conference on Rehabilitation Robotics, 2005, pp.465-468.

[27] D. Feil-Seifer, M. Matari, Towards the integration of socially assistive robots into the lives of children with ASD, International Conference on HumanRobot Interaction Workshop on Societal Impact: How Socially Accepted Robots Can be Integrated in our Society, 2009.

[28] H. Kozima, C. Nakagawa, Y. Yasuda, Interactive robots for Communications-care: a case study in autism therapy; in IEEE International Workshop on Robot and Human Interactive Communication (ROMAN), 2005, pp. 341-346.

[29] H. Kozima, C. Nakagawa, Y. Yasuda, Childrenrobot interaction: A pilot study in autism therapy. Prog Brain Res, 2007, vol. 164, pp. 385-407.

[30] C. Lathan, K. Boser, C. Safos, C., Frentz, K.Powers, Using cosmo's learning system (CLS) with children with autism. In proceedings of the International Conference on Technology-Based Learning with Disabilities, Dayton, 2007, pp. 3747.

[31] A. Tapus, J. Fasola, M. Matari, Socially assistive robots for individuals suffering from dementia. In: ACM/IEEE 3rd Human-Robot Interaction International Conference, Workshop on Robotic Helpers: User Interaction, Interfaces and Companions in Assistive and therapy Robotics, Amsterdam, The Netherlands, 2008.

[32] B. Scassellati, H. Admoni, M. Mataric, Robots for Use in Autism Research, Annual Review of Biomedical Engineering, 2012, vol. 14, pp. 27594.

[33] A. Solish, A. Perry, P. Minnes, Participation of children with and without disabilities in social, recreational and leisure activities, Journal of Applied Research in Intellectual Disabilities, 2010, vol. 23, pp. 226-236.

[34] M. Tennyson, D. Kuester, C. Nikolopoulos, Robotic agents used to help teach social skills to individuals with autism: The fourth generation, IEEE Symposium Series on Computational Intelligence, Orlando, 2014, pp. 42-48.

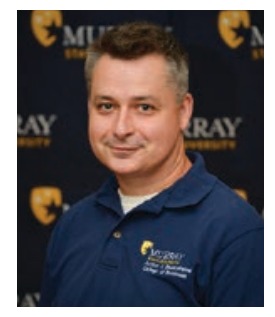

Dr. Matthew Tennyson received his $\mathrm{PhD}$ from Nova Southeastern University, MS from Bradley University, and BS from Rose-Hulman Institute of Technology. He is currently an Assistant Professor of Computer Science at Murray State University. Previously, he served as Lecturer at Bradley University and Director of CIS and MIS at Midstate College. His teaching and research interests include all aspects of software development and software engineering. Prior to academia, he developed electrical and embedded systems at Caterpillar, Inc., for various types of earth-moving machinery.

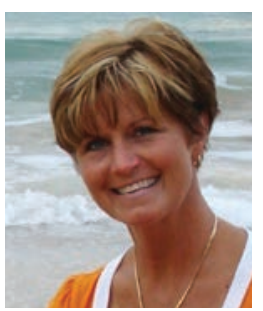

Dr. Deitra Kuester received her PhD from Purdue University and currently an Associate Professor of Teacher Education at Bradley University. Her teaching and scholarship centers on autism and related developmental and behavioral disabilities, multidisciplinary collaboration between STEM and secondary education fields of study, and teacher preparation. She has published and presented in over thirty refereed journals and international venues, coauthored book chapters, authored a book and co-authored a patent. She is the recipient of the Education and Health Sciences Outstanding Faculty Research Award for both 2012 and 2015 academic years.

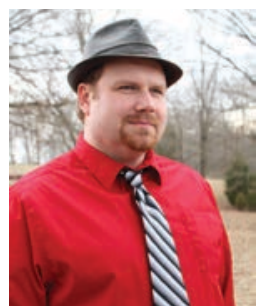

John Casteele is an undergraduate seeking a Bachelor of Science degree in Computer Science at Murray State University. The father of a child with autism, he hopes to one day work in the development of software and other computer-aided strategies for autism therapy. Prior to returning to school, Casteele enjoyed a 14-year career as a writer and editor for both print and online platforms.

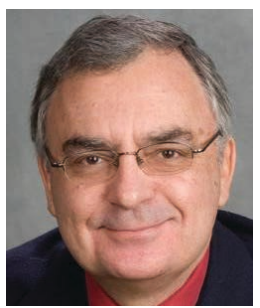

Dr. Christos Nikolopoulos received his $\mathrm{PhD}$ from Colorado State University and is currently a Professor of Computer Science at Bradley University. He has taught at Colorado State University, Louisiana State University, and Saginaw Valley State University. $\mathrm{He}$ is a recipient of the national Fulbright Senior Scholar Award. His teaching and research interests include artificial intelligence, data mining, and robotics. He is a textbook author and has published over 80 research papers in refereed journals and conference proceedings. He is coauthor of several US Patents and has been funded by over 1.5 million dollars in grants from various sponsors. 